\title{
Advances in the SMS design method for imaging optics
}

\author{
Wang $\operatorname{Lin}^{1}$, Pablo Benítez ${ }^{1,2}$, J.C.Miñano ${ }^{1,2}$, José Infante ${ }^{1}$, Guillermo Biot ${ }^{1}$ \\ 1. CEDINT, Universidad Politécnica de Madrid \\ 2. LPI-LLC, 2400 Lincoln Avenue, Altadena CA 91001, USA
}

\section{ABSTRACT:}

In this work, two SMS algorithms are presented for an objective design with different selected ray-bundles: three meridian ray-bundles $(3 \mathrm{M})$ and one meridian and two skew ray-bundles (1M-2S), the latter from pin hole point of view, provides a better sampling of the phase space. Results obtained with different algorithms will be compared.

\section{KEYWORDS}

SMS method, telephoto lens, Meridian ray-bundle, Skew ray-bundle, imaging system

\section{Introduction}

Traditional optical design methods are developed mainly from paraxial optics ${ }^{[1,2]}$. The limitation of classical methods is that designs with non-axial rays depend greatly on multi-parametric optimization techniques and the closeness of initial guess of the optical system to the optimum solution. If the initial design is far from the optimum point, it is most likely that either optimizer would fail to find useful solutions, or designers need to spend long hours to improve there initial guesses.

The Simultaneous Multiple Surfaces (SMS) method is a direct construction method for selected ray-bundles, meridian or skew ray-bundles. The SMS method was developed as a design method in Non-imaging Optics during the $90 \mathrm{~s}^{[3-7]}$, where the imaging formation is not required. A famous theorem in Non-imaging optics is the edge-ray theorem, which states that all rays can be transferred to the target, if the edge-rays in phase space are transferred to the target. Therefore, SMS method in the beginning only used edge-rays from light sources like Sun or LED. With the development of SMS methods from 2 surfaces to 4 surfaces, it has been found that by sampling in the phase space properly with more ray-bundles $(\mathrm{N}>2)$ and designing with SMS method for the selected ray-bundles, image quality is greatly improved. Recently research shows that SMS method is superior to aplanatic design method in controlling non-paraxial rays ${ }^{[9,10]}$.

There have already been several applications of SMS methods to imaging optics ${ }^{[8-14]}$, especially in Ref 12,2 rotational mirrors were design with 2 symmetric skew ray-bundles. In this work, the SMS method is extended by designing 1 meridian ray-bundle and 2 symmetric skew ray-bundles(SMS 1M-2S) with 3 rotational surfaces. As a comparison of difference in phase space sampling, another design is realized with 3 meridian ray-bundles (SMS 3M).

\section{Sampling of phase space}

In a rotational symmetric system, SMS design procedure involves simultaneous calculation of $\mathrm{N}$ rotational surfaces for given $\mathrm{N}$ uni-parametric ray bundles. In Fig 1a, yz plane is considered as meridian plane. An object is seen by a pin hole, which locates at the y' axis of the entrance pupil plane. The pin-hole can also be moved freely along that y' axis. In Fig 1a, 3 object points $\mathrm{M}_{1}, \mathrm{M}_{2}$ and $\mathrm{M}_{3}$ are placed equal-spaced along y axis. Therefore, rays coming from those 3 object points to the pin hole are all meridian rays. By moving the position of the pin hole along the y' axis, 3 meridian ray-bundles (3M) from the 3 meridian object points can be defined. In Fig $1 \mathrm{~b}$, object point $\mathrm{M}_{1}$ is placed on $\mathrm{y}$-axis, and another 2 object points $S_{1}$ and $S_{2}$ are placed symmetrically about y axis. Ray from $\mathrm{M}_{1}$ to pin hole is a meridian ray and 
rays from object points $S_{1}$ and $S_{2}$ are skew rays. Moving pin-hole position will therefore define 1 meridian ray-bundle and 2 symmetric skew ray-bundles (1M-2S)In fact, there are many other ways to define skew ray-bundles, based on practical considerations for each application ${ }^{[12,13]}$.
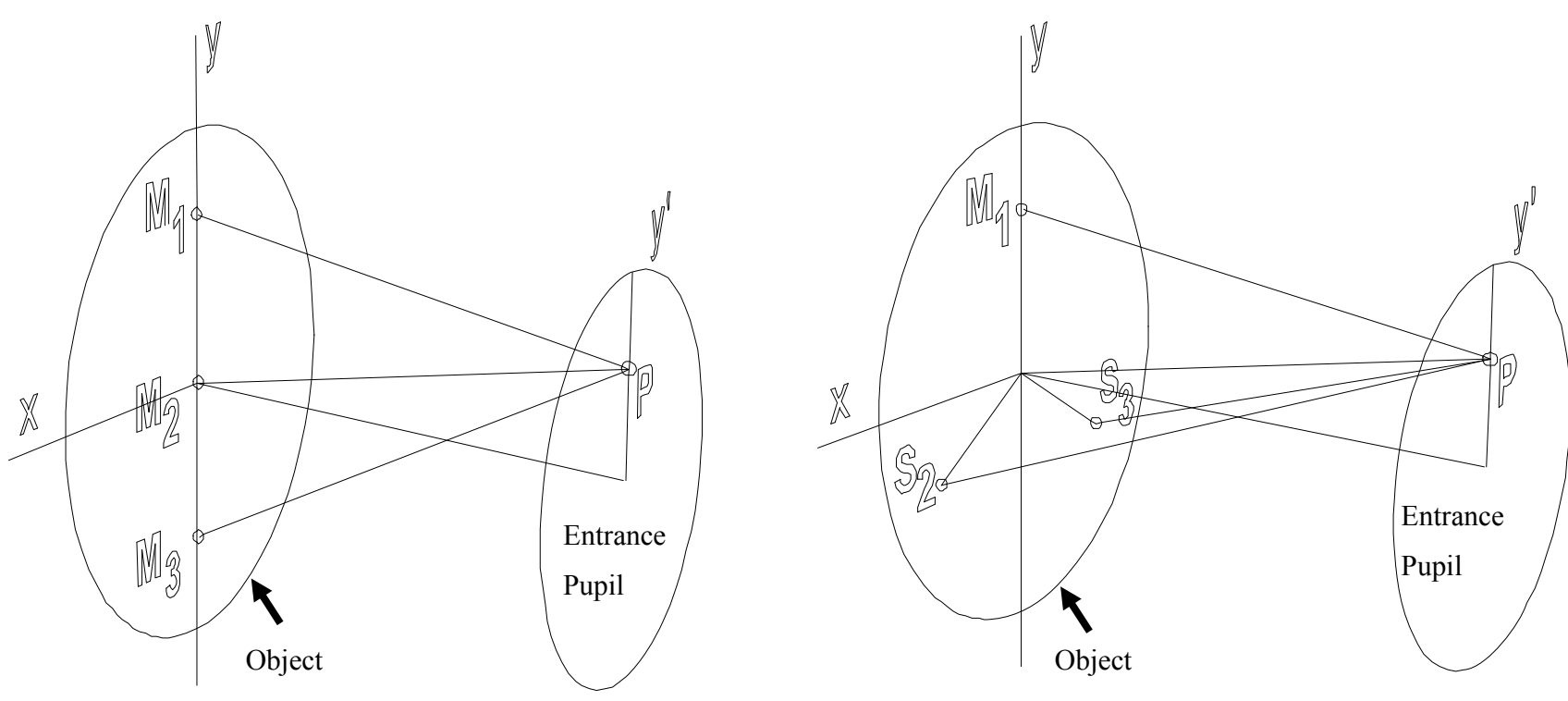

Fig 1. Samplings in phase space for a rectangular object.

a) 3 meridian ray-bundles sampling

b) 1 meridian 2 skew ray-bundles sampling

Obviously from the pin-hole point of view, 1M-2S ray-bundles provide a better sampling of all rays in the phase space than $3 \mathrm{M}$ ray-bundles.

\section{SMS 1M-2S Wave-front criteria}

Detailed SMS 2S algorithm can be found in ref [12]. Different from SMS 2S algorithm where initial curves were not necessary in the design, SMS 1M-2S algorithm needs initial curves for further SMS construction. Similar to SMS 3M algorithm ${ }^{[14]}$ where a 2D wave-front criteria for meridian ray-bundles is proposed, a wave-front criteria is presented here for 1 meridian and 2 skew ray-bundles, as shown in Fig 2.3 object points $M_{1}, S_{1}, S_{2}$ and 3 image points $M_{1}{ }^{\prime}, S_{1}$ ', $S_{2}$ ' are placed equal-spaced in object and image plane. $V_{1}, V_{2}, V_{3}$ are 3 central vertexes of initial curves, which can be defined loosely from mechanical or optical requirements. 3 chief rays from 3 object points all pass through point $\mathrm{V}_{2}$, center of Curve 2; therefore they are all meridian rays. Skew ray-bundles around chief ray of $S_{1}$ and $S_{2}$ situate in a skewed plane which has an angle $\alpha$ with meridian plane $(\alpha \neq 0)$. In this way, selected ray-bundles for point $S_{1}$ and $S_{2}$ are all skew rays 
except the first ray.

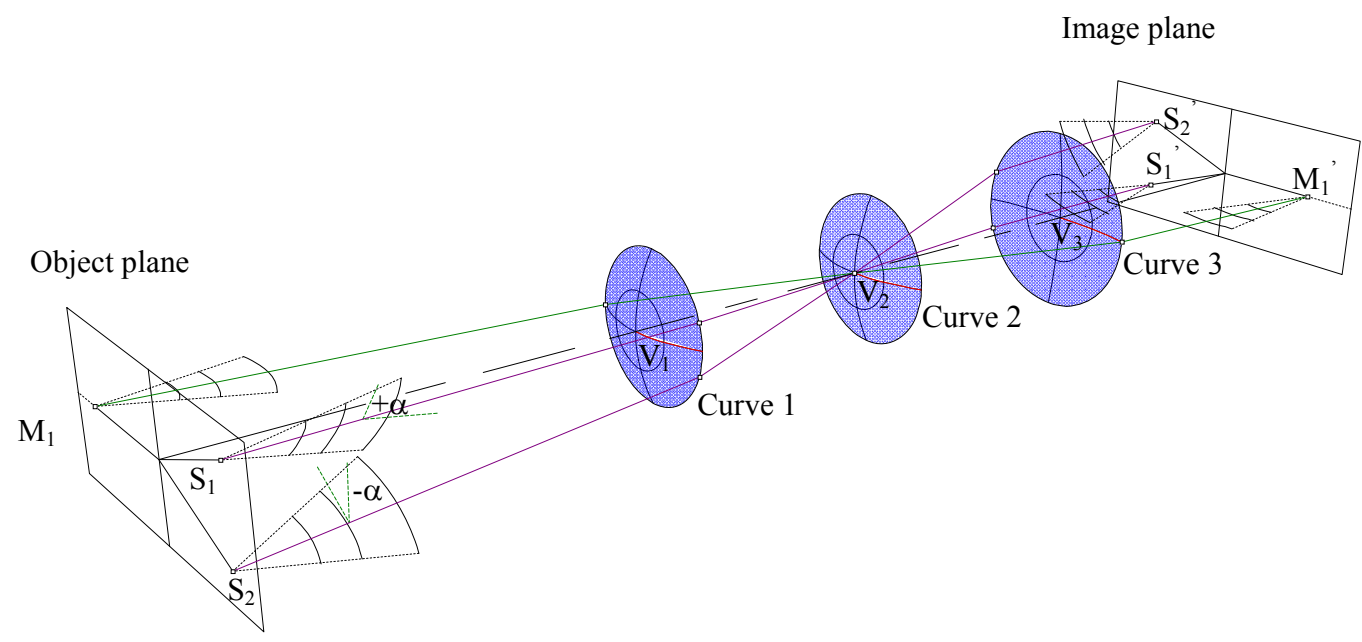

Fig 2. Wave-front criteria for $1 \mathrm{M}-2 \mathrm{~S}$ initial curves

The wave-front criteria requires that wave-fronts formed by selected ray-bundles, close to chief rays in object space, should be transformed into wave-fronts of its corresponding image points by passing through this rotational optical system with generalized ray-tracing ${ }^{[15]}$. Therefore, central rotational surfaces perfectly image object points into image points for selected ray-bundles.

Once initial curves are obtained, SMS construction can be started in the following order:

1. Meridian ray-bundle is used to construct Curve 3

2. Skew ray-bundles are used to construct Curve 1 and Curve 2 at the same time ${ }^{[12]}$

3. Repeat the above process.

Detailed 3M algorithm is presented in Ref [14].

\section{Tele-photo lens designs with SMS 1M-2S and SMS 3M algorithms}

Parameters of objective lens are listed in table 1. Artificial material with 1.5 refractive index is used for both designs to compare performance of initial monochromatic designs obtained by SMS methods.

Table 1. optical parameters

\begin{tabular}{|l|l|} 
Focal length & $9 \mathrm{~mm}$ \\
\hline F\# & 2 \\
\hline Field of view & $\pm 15^{0}$ \\
\hline Refractive index & 1.5 (artificial material) \\
\hline Lens' surfaces & $\begin{array}{l}1 \text { Frontal planar surface } \\
3 \text { aspherical surfaces }\end{array}$ \\
\hline
\end{tabular}

In Fig 3a, 2 lenses are going to be designed by SMS algorithms for object angles $\pm 8.84^{0}$. The first refractive surface is planar, and other 3 aspherical surfaces are calculated by SMS methods. Central vertices of the 4 optical surfaces are taken approximately from the previous work on SMS 4M algorithms ${ }^{[9]}$. Fig 3b shows RMS spot diameter (RMS 3D) results with respect to different object angles. In these initial designs by SMS 3M algorithm and SMS 1M-2S algorithm, the central vertex of second refraction surface $\mathrm{V}_{2}$ takes the same initial value $\left(\mathrm{d}_{0}=30 \mathrm{~mm}\right.$ from image plane). 


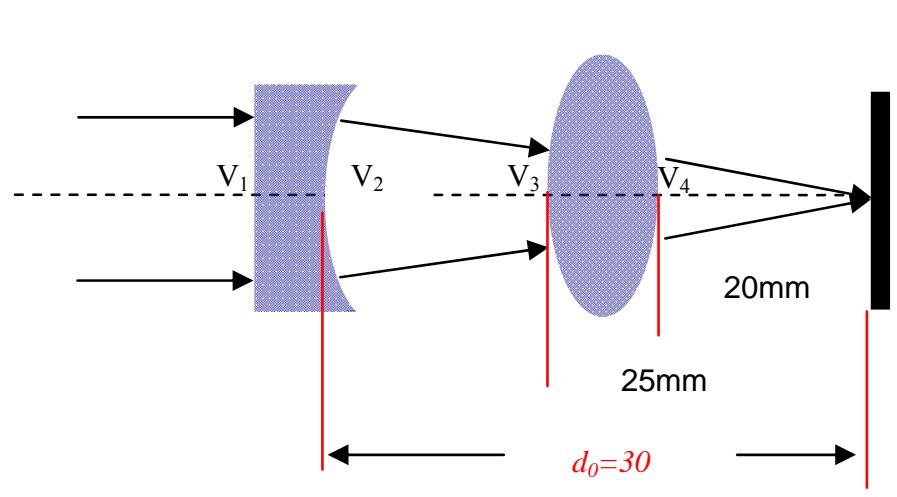

a) sketch of the desing

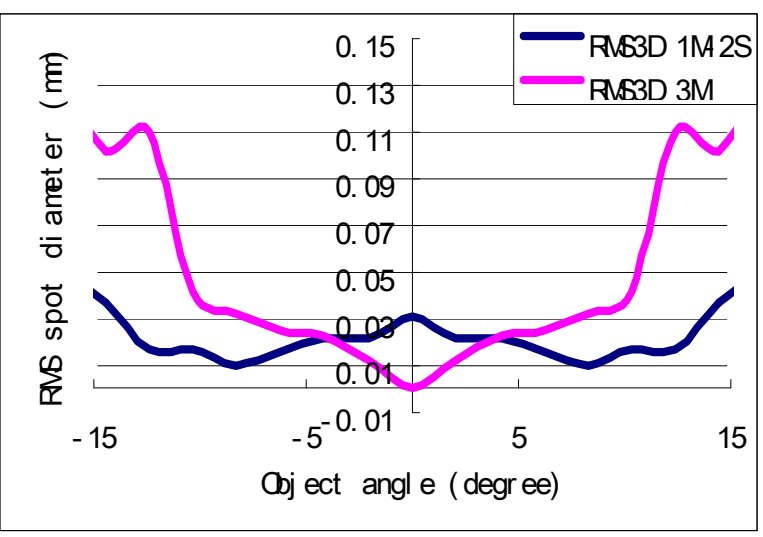

b) RMS 3D results

Fig 3. sketch of design and results by SMS 3M and SMS 1M-2S algorithms

Then, the vertex position of the second refractive surface $\mathrm{V}_{2}$ is used as one degree of freedom to minimize largest RMS spot diameter(RMS 3D) over all fields of view. During this one parameter optimization, SMS 1M-2S algorithm is repeated for each different vertex position of last refractive surface. A similar design with SMS 3M algorithm is realized to compare different strategies of phase space sampling. The central vertex of curve $\mathrm{R}_{2}$ is also used to minimize RMS $3 \mathrm{D}$ over all fields of view.

Fig 4 shows that: different optimum vertex positions are found for SMS 1M-2S design (37mm from image plane) and SMS 3M design (39mm from image plane); A close comparison between curve shapes of $\mathrm{R}_{3}$ and $\mathrm{R}_{4}$ showed in Fig $4 \mathrm{c}$ also demonstrates that the optimum designs from different selection of ray-bundles and SMS algorithms will lead to a similar curve shapes.

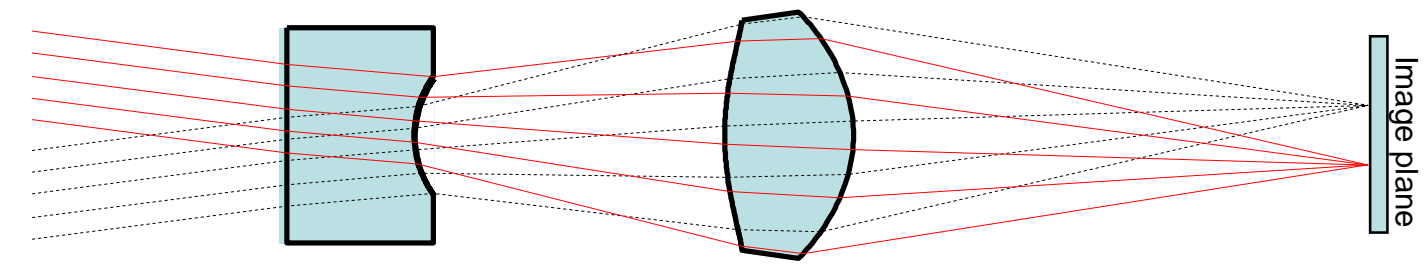

a) SMS $1 M-2 S$ design

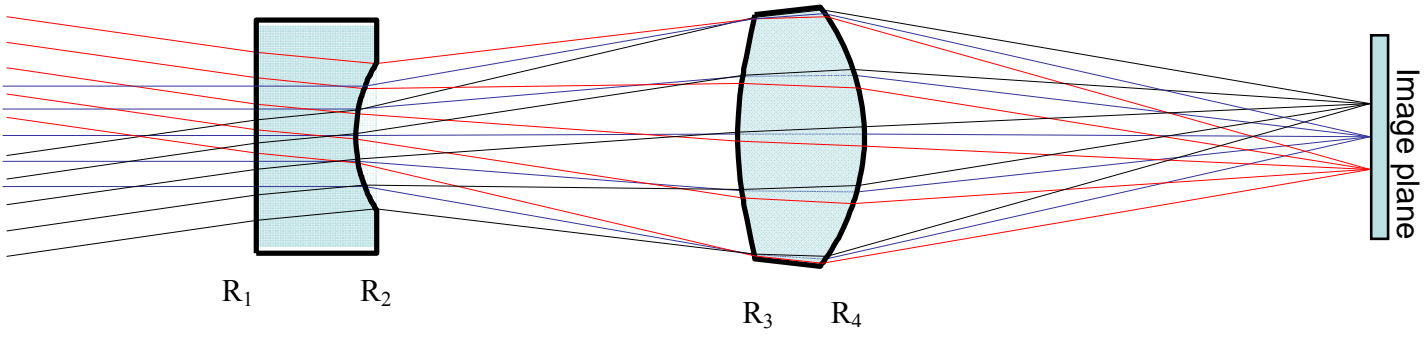

b) SMS 3M design

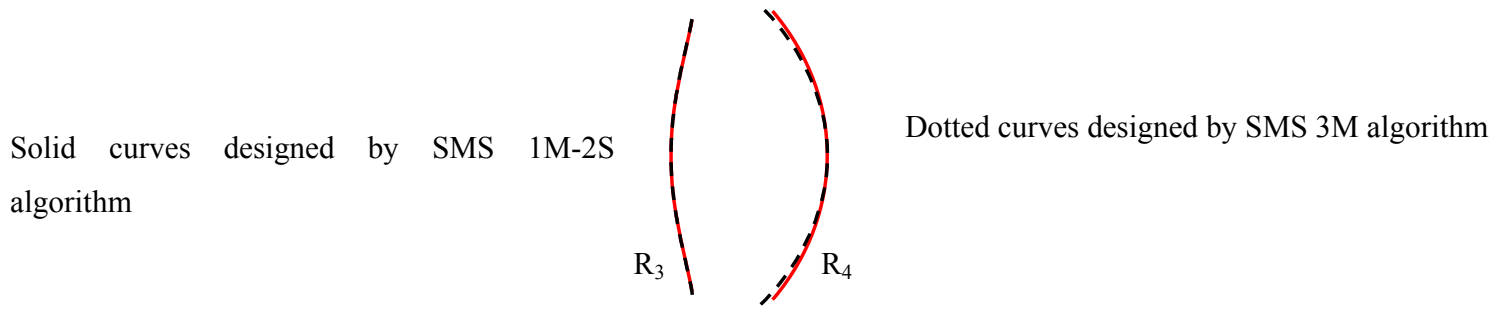

c) Comparison of $\mathrm{R}_{3}$ and $\mathrm{R}_{4}$

Fig 4. Designs obtained by optimization with SMS algorithms 
Comparing RMS3D(RMS spot diameter calculated by ray-tracing both meridian rays and skew rays) results in Fig 5 , Fig 5, it can be seen that RMS distribution of SMS 1M-2S design is in general more uniform than that of SMS 3M design. This could be understood as that, by designing ray-bundles which distribute more uniformly in phase space with SMS 1S-2M algorithm, the power of control over other fields of view is very similar. Also, the power of control over large fields of views ( $>10$ degree) is very similar for both designs. However, due to lack of control of on-axis rays, SMS $1 \mathrm{M}-2 \mathrm{~S}$ algorithm is less effective in minimization of RMS spot size for the central field of view. Therefore, SMS $3 \mathrm{M}$ algorithm provides a bit better initial design than SMS 1M-2S algorithm in this objective design.

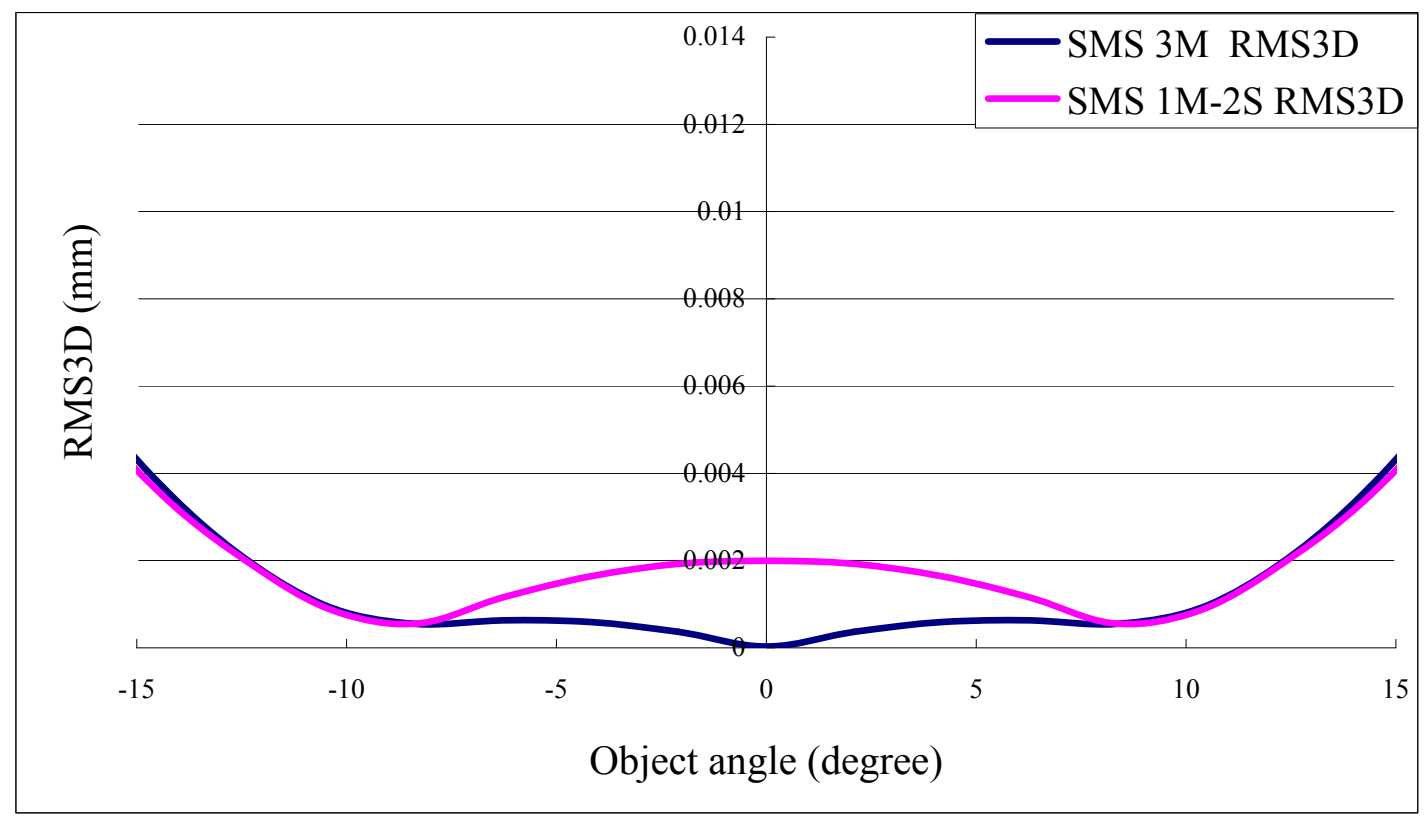

Fig 5. Comparison of RMS spot diameters

To further understand the power of control over skew rays in both designs, distribution of RMS spot diameters over different skew ray-bundles ( $\operatorname{RMS}(\alpha, \beta))$ are calculated, as shown in Fig 6. " $r$ " indicates the radial position of object point on the object plane; in case the object plane at infinity, object angle " $\alpha$ " is used instead. " $\beta$ " represents the radial angle about axis $\mathrm{Y}$ in the entrance pupil.

\section{Object}

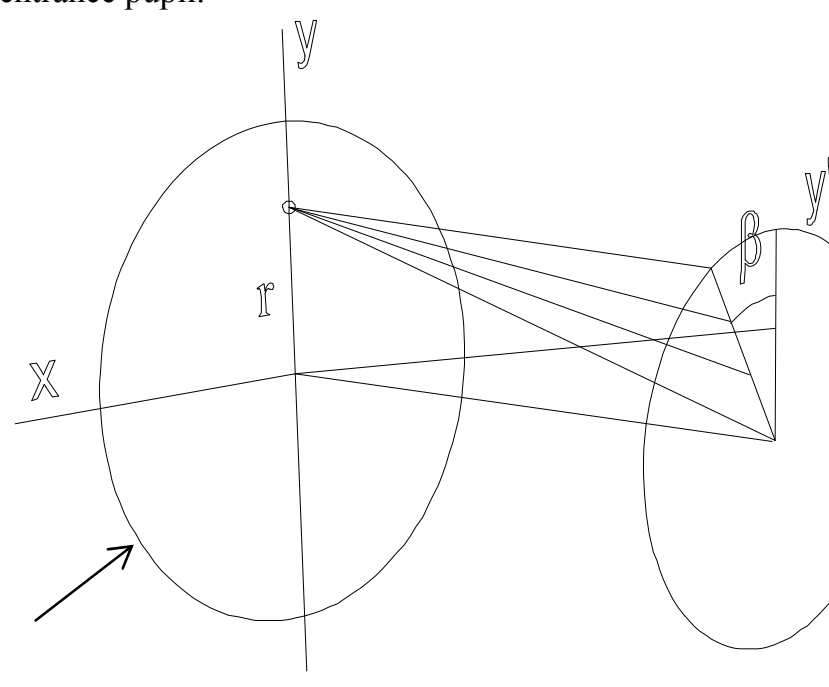

Fig 6. $\operatorname{RMS}(\alpha, \beta)$ distribution

\section{Entrance pupil}




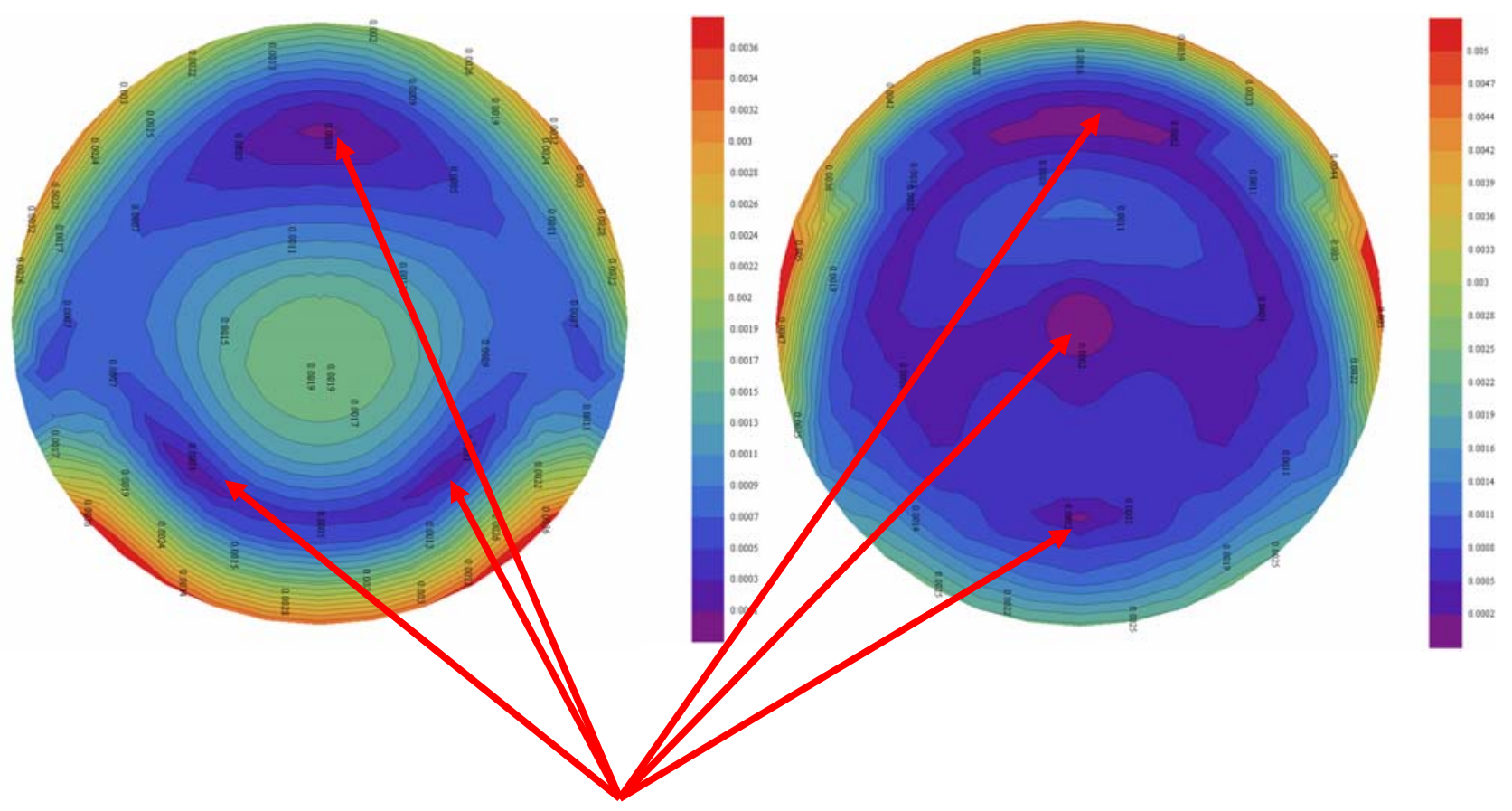

SMS design points

a) SMS $1 \mathrm{M}-2 \mathrm{~S}$

b) SMS 3M

Fig 6. $\operatorname{RMS}(\alpha, \beta)$ results of both designs

It can be seen from results in Fig 6 that in both $\operatorname{RMS}(\alpha, \beta)$ distributions, there are 3 points with low RMS spot diameters $(<0.2 \mu \mathrm{m})$; therefore both algorithms have successfully control the designed ray-bundles. Also, central RMS distribution of SMS 3M design has smaller values than that of SMS 1M-2S design. Integral of RMS $(\alpha, \beta)$ over radial angle $\beta$ will lead to results shown in Fig 4.

\section{Conclusion}

In this work, we have developed a SMS 1M-2S algorithm with wave-front criteria for initial curves selection, and applied the algorithm to a monochromatic objective design. By the vertex optimization of the second refractive surface, which is a one parameter optimization, largest RMS3D in all fields of view has dropped to $4 \mu \mathrm{m}$ for both designs. Results have shown that by selecting ray-bundles which spread uniformly in phase space, SMS 1M-2S design has a more uniform RMS 3D distribution after optimization. However compared to SMS 3M design, it still has less control of RMS spot size over all fields of view, especially the center field of view.

\section{Acknowledgement}

Authors thank the Spanish Ministries MCINN (ENGINEERING METAMATERIALS: CSD2008-00066, DEFFIO: TEC2008-03773, SIGMASOLES: PSS-440000-2009-30), MITYC (ECOLUX: TSI-020100-2010-1131, SEM: TSI-020302-2010-65) and the Madrid Regional Government (SPIR: 50/2010O.23/12/09,TIC2010 and O-PRO: PIE/209/2010) and UPM (Q090935C59) for the support given in the preparation of the present work. 


\section{Reference}

[1] W. J. Smith, Modern Optical Engineering, 3rd ed. (McGraw-Hill, 2000).

[2] R. E. Fisher and B. Tadic-Galeb, Optical System Design (McGraw-Hill, 2000).

[3] Chapter 8 in R.Winston, J.C. Miñano, P. P. Benítez, with contributions of N. Shatz, J. Bortz, "Nonimaging Optics", Academic Press Elsevier (2004).

[4] Chaves, J., [Introduction to Nonimaging Optics], CRC Press, (2008).

[5] Pablo Benítez, Juan C. Miñano, José Blen, Rubén Mohedano, Júlio Chaves, Oliver Dross, Maikel Hernández, and Waqidi Falicoff, "Simultaneous multiple surface optical design method in three dimensions," Opt. Eng. 43, 1489 (2004). [6] Dross, O., Cvetkovic, A., Chaves, J., Benítez, P., Miñano, J.C., "LED Headlight Architecture that creates a High Quality Beam Pattern independent of LED Shortcomings", Proc. SPIE 5942, 126-135 (2005).

[7] Miñano, J.C., González, J.C., "New method of design of nonimaging concentrators", Appl. Opt. 31, 3051-3060 (1992).

[8] P. Benítez and J. C. Miñano, "Ultra high-numerical-aperture imaging concentrator," J. Opt. Soc. Am. A, Vol 14 (1997).

[9] J. C. Miñano, P. Benítez, Wang Lin, José Infante, Fernando Muñoz, Asunción Santamaría

"An application of the SMS method for imaging designs," Optical Express, Vol 17, No. 26, p. 24036 (2009).

[10] Miñano, J.C., Benítez, P., Wang Lin,, Muñoz, F., Infante, J., Santamaría, A., "Overview of the SMS design method applied to imaging optics", Proc. SPIE 7429, 74290C (2009).

[11] Jose M. Infante Herrero, Fernando Muñoz, Pablo Benitez, Juan Carlos Miñano, Wang Lin,

Juan Vilaplana, Guillermo Biot, Marta de la Fuente "Novel fast catadioptric objective with wide field of view", Proc.

SPIE 7787, 778704 (2010)

[12] F. Muñoz, P. Benítez, and J. C. Miñano "High-order aspherics: the SMS nonimaging design method applied to imaging optics", Proc. SPIE 7061, 70610G (2008).

[13] Fernando Muñoz Doctoral Disertation "Sistemas ópticos avanzados de gran compactibiliad con aplicaciones en formación de imagen y en iluminación" UPM, Madrid (2004)

[14] Wang Lin, Pablo Benitez, Juan Carlos Miñano,Jose M. Infante Herrero, Marta de la Fuente, Guillermo Biot, "Ultra-compact SWIR telephoto lens design with SMS method," Proc. SPIE 8129, 812616(2011).

[15] Orestes N. Stavroudis, "The mathematics of geometrical and physical optics", Wiley-VCH (2006). 